Cytogenet Genome Res 1996;73:348

\title{
Subject Index, Vol. 73, 1996
}

This index does not contain items of the reports of the Chromosome Mapping Workshops 2, 12, and $\mathrm{Y}$ and the Report of the International Meeting on Human Chromosome 12 Genes in Cancer 1995 which are published in this volume.

Animals

babirusa (Babyrousa babyroussa)

203 catfish (Ictalurus punctatus) 282 cattle 184,229 dog 140 duiker antelope (Cephalophinae:Bovidae) 116 eel (Anguilla anguilla) 149 lungfish (Neoceratodus forsteri) 187 mouse 86,279, 286, 308 nonhuman primates 92 rat 79,209,228,286 swine 164,194,274 vole (Microtus agrestis) 171 water rat (Nectomys) 190

Banded chromosome analysis babirousa(RBP,QFQ) 203 cattle(T) $229 \operatorname{dog}(\mathrm{R}, \mathrm{G}, \mathrm{GTG}) 140$ duiker antelope (GTG, C) 116 eel(DAPI,C) 149 human(AD/DAPI) 104,250 human(DAPI) 99,134,295 human(G) 157,168,334 human(G,GTG) 322 human(GTP) 123 human(QFQ) 92 human(R) 97, 114, 130, 137, 153, 214,218,250,312 lungfish $\mathrm{G}, \mathrm{C}) 187$ mouse(C) 86 mouse(R) 286 nonhuman primates(QFQ) $92 \operatorname{rat}(\mathrm{G}) 209 \operatorname{rat}(\mathrm{Q}) 79 \operatorname{rat}(\mathrm{R}) 286 \operatorname{swine}(\mathrm{G})$ 164,194 vole(G,C) 171 water $\operatorname{rat}(\mathrm{G}, \mathrm{C}) 190$

Cell cycle

DNA, sex chromosomes and NORs 108

UBF, rDNA and NORs 274 Chromosome aberration aneuploidy in sperm 300

distal trisomy 14123

gene amplification 325

point mutation analysis 343

translocation 179,279,325 Chromosome mapping workshops

chromosome 2 report 255

chromosome 12 report 1

chromosome 12 genes in cancer 25

Y chromosome report 33 Comparative gene mapping antelope 116

cattle 116,184

mouse $97,153,157,279,286,312$

nonhuman primates 92

rat $79,209,286,312$

swine 164

Evolution

bovid X chromosome 116

FCGR1 genes 157

human chromosome 992

lungfish 187 
Microtus gnomosomal heterochro-

matin 171 pig chromosomes 3 and 6203

Flow cytometry

human marker chromosome 123

Fluorescent in situ hybridization (FISH) archival G-banded slides 322 comparative genome painting 116 localization of rDNA 108,149,274,

290 multi-color PRINS 300 probe less than 500 bp 297

Gene mapping cattle

ADCYAP1, CDH2, GRP,

MCR2, PAI2, TTR, TYMS 184 eel

rDNA genes 149 human

ASCL2 312

CDC46 317

CKS1, CKS2 250

CLAPS3 214

D20S756 315

DIFF6 224

FCGR1A, FCGR1B, FCGR1C 157

FCGRT 97

FMR1L2 240

GCN5L1 134

GRSF1 295

HOXA@,HOXB@,HOXC@,HOXD@114

IGHEP2 92

MEF2A, MEF2AP 244

NF1 homologous loci 334

PTPRE 168

PTPRN 145

RABL 137

RP12 81

RPD3L1 130

RSC1A1 341

SEC14L 218

SKP1A, SKP1B, SKP2 104

SLC5A3 77

SRD5A2 304

SSX3 179

TCTEL1 153

UQCRB 297

WARS 99 mouse

Scp2, scp2-ps1, scp2-ps2 279

Sdfl 286 nonhuman primates

IGHEP2 92 rat

KrtlO1 209

Pdnpno 228

Tbxa2r 79 swine 
EPO, IL5, MDH2, TCRB, ZP3 164

Heterochromatin

C-bands and LRR copy number 86

methylation in vole $\mathrm{X}$ chromosome 171

protein 1 localization 308 Human disorders

Charcot-Marie-Tooth disease 343

Down syndrome 77

familial juvenile nephronophthisis 235

male pseudohermaphroditism 304

prostate cancer 304

psychodevelopmental abnormalities 123

retinitis pigmentosa 81

rhabdomyosarcoma 325

synovial sarcoma 179

tumor susceptibility gene 250

Type 1 neurofibromatosis 334 Hybrids

hamster-bovine 184

human-rodent 99, 157

pig-rodent mapping panel 164,194

radiation hybrid panel 240

vole-mouse 171

Interspecific backcross mapping mouse 286

Karyotype

babirusa compared with pig 203 bovine T and R banded 229 canine 140 lungfish 187 Nectomys

190

Linkage

chromosome 1 pericentric linkage

group $157 \mathrm{GPI}$ and sex determination in

catfish 282 haplotype analysis of RP12 on lq

81 LHRH, ADRB3, HGL on 8p 331 SRD5A2 and 2p markers 304 WARS and 14q 99

Meiosis

detection of nondisjunction 300 Nectomys hybrids 190 spatial distribution of sex chromosome and nucleolus 108

Physical mapping

marker near 20pter 315 NPH1 region on 2 q 13235 integrated with genetic map 17p 240

Repeats

$\alpha$-sat DNA associated with NF1 334 centromere in marker chromosome

123 copy number of LRR 86 gene specific STRPs 331 mid-size repeat in large repeat

element 171 polymorphic microsatellite at $2 \mathrm{q} 35$

145 VNTR polymorphic marker 218

Ribosomal genes

human sperm nucleoli 290

gene clusters in eels 149

NORs and DNA binding protein 274

spatial relationship with sex chromosomes 108

Sequence 
ASCL2 312

CDC46 317

CKS2 250

CLAPS3 214

DIFF6 244

GCN5L1 134

MEF2a, MEF2AP 244

PTPRN 145

RABL 137

RPD3L1 130

SEC14L 218

SSX1, SSX2, SSX3 179

TCTEL1 153

telomer marker 315 STSs

bovine 184

ESTs at $15 \mathrm{q} 26$ identified 244

order in chromosome $2 \mathrm{q} 13$ region 235

order on chromosome $17 \mathrm{p} 240$

VN02 marker 77

Techniques

CISS on archival G-banded slides

322 PCR and sequencing archive slide

DNA 343 sequential multicolor PRINS 300

348

Cytogenet Cell Genet, Vol. 73, 1996 